\title{
Effect of IAA on in vitro growth and colonization of Nostoc in plant roots
}

\author{
Anwar Hussain ${ }^{1}$ *, Syed T. Shah ${ }^{2}$, Hazir Rahman ${ }^{3}$, Muhammad Irshad $^{1}$ and Amjad Iqbal ${ }^{4}$ \\ ${ }^{1}$ Department of Botany, University College of Science Shankar Campus, Abdul Wali Khan University Mardan, Mardan, Pakistan \\ 2 Nuclear Institute for Food and Agriculture, Tarnab Peshawar, Pakistan \\ ${ }^{3}$ Department of Microbiology, Kohat University of Science and Technology, Kohat, Pakistan \\ ${ }^{4}$ Department of Food Science, University College of Science Shankar Campus, Abdul Wali Khan University Mardan, Mardan, Pakistan
}

Edited by:

Erh-Min Lai, Academia Sinica, Taiwan

Reviewed by:

Jeff H. Chang, Oregon State

University, USA

Chi-Te Liu, National Taiwan University,

Taiwan

\section{*Correspondence:}

Anwar Hussain, Department of

Botany, University College of Science

Shankar Campus, Abdul Wali Khan

University Mardan, Mardan 23200,

Khyber Pakhtunkhwa, Pakistan

e-mail:drhussain@awkum.edu.pk
Nostoc is widely known for its ability to fix atmospheric nitrogen and the establishment of symbiotic relationship with a wide range of plants from various taxonomic groups. Several strains of Nostoc produce phytohormones that promote growth of its plant partners. Nostoc OS-1 was therefore selected for study because of the presence of putative ipdC gene that encodes a key enzyme to produce Indole-3-acetic acid (IAA). The results indicated that both cellular and released IAA was found high with increasing incubation time and reached to a peak value (i.e., $21 \mathrm{pmol} \mathrm{mg}^{-1} \mathrm{ch}-a$ ) on the third week as determined by UPLC-ESI-MS/MS. Also the Nostoc OS-1 strain efficiently colonized the roots and promoted the growth of rice as well as wheat under axenic conditions and induced ipdC gene that suggested the possible involvement of IAA in these phenotypes. To confirm the impact of IAA on root colonization efficiency and plant promoting phenotypes of Nostoc OS-1, an ipdC knockout mutant was generated by homologous recombinant method. The amount of releasing IAA, in vitro growth, root colonization, and plant promoting efficiency of the ipdC knockout mutant was observed significantly lower than wild type strain under axenic conditions. Importantly, these phenotypes were restored to wild-type levels when the ipdC knockout mutant was complemented with wild type ipdC gene. These results together suggested that ipdC and/or synthesized IAA of Nostoc OS-1 is required for its efficient root colonization and plant promoting activity.

Keywords: Nostoc, putative ipdC, endophyte, IAA, phytostimulation, knockout

\section{INTRODUCTION}

Co-existence has evolved in several forms of living organisms for successful life on the planet earth. Cyanobacteria are a group of the most ancient oxygenic phototrophic organisms that served the sole source of oxygen in the earth's atmosphere about 2.5 billion years back (Buick, 2008). Nostoc is a genus of nitrogen fixing cyanobacteria found in a number of environmental niches. Colonies of Nostoc are composed of filaments of moniliform cells embedded in a gelatinous sheath. Members of this genus associate symbiotically with diverse taxonomic groups of organisms (such as algae, Bryophytes, Pteridophytes, gymnosperms and angiosperms), benefiting their hosts with nitrogen in the form of nitrates (Rai and Bergman, 2000). In nature, Nostoc may grow as epiphytes (Myllys et al., 2007), endophytes or symbionts, making beneficial association (Pankratova et al., 2008; Olsson et al., 2012). In addition to nitrogen and carbon fixation (Adams and Duggan, 2012), symbiotic Nostoc can also release phytohormones Indole-3-acetic acid (IAA and cytokinins), which improve plant vigor (Hussain et al., 2010).

Production of IAA by Nostoc (Sergeeva et al., 2002) and its involvement in plant-microbe signaling has been known for years (Berg, 2009). Microbes are believed to reduce cell wall integrity of the host cell, colonize there and induce plant roots to release more sugars (Boopathi et al., 2013). Aditionally, IAA may de-repress auxin signaling in plants, helping the bacteria to evade the plant defense system (Zhang et al., 2007). Inhibition of IAA transport or mutation in auxin biosynthesis gene on the other hand reduces root colonization (Karabaghli-Degron et al., 1998; Bossuyt et al., 2012). However, the role of this hormone in root colonization by cyanobacteria is less obvious and has not been determined.

Indole-3-acetic acid can be synthesized by both tryptophan dependant and tryptophan independent pathways, but the former is a usual one while the latter is bioenergetically costly (Prinsen et al., 1993; Akashi and Gojobori, 2002). Prokaryotes biosynthesize IAA from tryptophan through one of the three major pathways, out of the three, plant friendly bacteria can synthesize it via the intermediate, indole-3-pyruvate (Bruto et al., 2014). The first step in the indolepyruvate pathway is the transfer of the amino group from tryptophan to $\alpha$-ketoglutarate by an enzyme aminotransferase, yielding indole-3-pyruvate and L-glutamate. Indole-3-pyruvate is then converted to indole-3-acetaldehyde by a rate limiting enzyme, indolepyruvate decarboxylase (IPDC; Patten etal., 2013). This enzyme belongs to a family of thiamine diphosphate-dependent decarboxylases which may catalyze degradation (indolepyruvate and phenylpyruvate decarboxylases), production of alcohols (pyruvate decarboxylases) or amino acid synthesis (acetolactate synthases; Schütz et al., 2003).

The biological role of IPDC has been confirmed in several bacteria, including Enterobacter cloacae (Koga et al., 1991), Azospirillum brasilense (Costacurta et al., 1994) and Pantoeaag glomerans 
(Brandl and Lindow, 1997). This enzyme is encoded by ipdC gene reported in the above mentioned and several other bacteria. Whereas, in cyanobacteria, no ipdC gene has been experimentally characterized till date but a homolog of ipdC known as thiamine pyrophosphate dependent decarboxylase has been found in the genome of Nostoc PCC 73102 (Sergeeva et al., 2002). The idea that cyanobacteria can synthesize IAA via indolepyruvate pathway was supported by the findings of Sergeeva et al. (2002). Their results demonstrated that various strains of Nostoc produced high levels of IAA, when grown on tryptophan rich media. In the current study, we focused on the ability of endophytic Nostoc to colonize rice and wheat plants under laboratory conditions. Putative IAA biosynthesis gene ipdC was knocked out form Nostoc to investigate its impact on root colonization potential of the Nostoc.

\section{METHODOLOGY}

\section{ISOLATION OF ENDOPHYTIC Nostoc STRAINS}

Wheat and rice plants were uprooted in the month of April and August, respectively, and brought to the laboratory in polythene bags. Soil particles were washed from the root segments $(0.5 \mathrm{~cm}$ in length) of the selected plants with running water. The root segments were surface sterilized by sequential dipping in $70 \%$ (v/v) ethanol for $5 \mathrm{~min}$, and sodium hypochlorite solution $(0.9 \%$, $\mathrm{w} / \mathrm{v}$ ) for $20 \mathrm{~min}$. The sterilized root samples were again rinsed three times with sterile distilled water to remove traces of sodium hypochlorite and were injured with sterile blade. The root segments were then plated on BG 11 medium (Barker and Tagu, 2000) containing cyclohexamide $\left(400 \mathrm{mg} \mathrm{L}^{-1}\right)$ for 3 weeks at $25^{\circ} \mathrm{C}$ and photoperiod of $8 \mathrm{~h}$ light and $16 \mathrm{~h}$ dark. These culture conditions were used throughout this report, unless otherwise mentioned.

After 3 weeks of incubation, the endophytic cyanobacterial colonies were picked from the mixed cultures and enriched in liquid BG11 media $(20 \mathrm{~mL})$ to obtain a sufficient biomass. Biomass were harvested from the cultures by centrifugation $(12000 \mathrm{rpm}$ for $2 \mathrm{~min}$ ) and re-suspended in $50 \mathrm{~mL} \mathrm{BG11} \mathrm{medium,} \mathrm{vortexed,}$ and spread on plates of BG11 containing cyclohexamide (400 mg $\mathrm{L}^{-1}$ ). The purity of the colonies was checked by light microscopy and pure colonies were transferred to new BG11 plates incubated under the conditions mentioned above.

To validate the procedure of surface sterilization: (i) surface

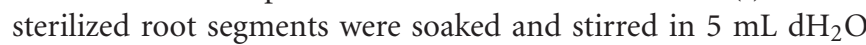
for $1 \mathrm{~min}$. Two hundred microliter suspension was then plated on BG11 agar plates and microbial growth was checked on plates incubated at $25^{\circ} \mathrm{C}$ under conditions mentioned earlier; (ii) the isolated cyanobacterial cultures subjected to the same procedure of surface sterilization, were inoculated on BG11 medium at $25^{\circ} \mathrm{C}$ for 3 weeks and recorded for cyanobacterial vitality.

\section{SCREENING OF Nostoc STRAINS FOR IAA}

Method of Hussain et al. (2010) was followed to screen different Nostoc cultures for IAA production. Simply, the strains were grown for 3 weeks in liquid BG11 media as mentioned earlier with the addition that $1 \mathrm{~mL}$ of the supernatent from cyanobacterial culture was mixed with $2 \mathrm{~mL}$ Salkowski reagent. The mixture was allowed to stand for $30 \mathrm{~min}$ in dark at room temperature and the absorbance were then measured at $540 \mathrm{~nm}$ using PerkinElmer
Lambda 25 spectrophotometer. The experiment was repeated three times with three replicates for each isolate.

\section{IDENTIFICATION OF THE SELECTED STRAIN}

To identify the selected strain, genomic DNA was isolated from the cultures grown for 15 days by UltraClean Soil DNA isolation Kit TM (Mo Bio Laboratories, Carlsbad, CA, USA) following the manufacturer's instructions. The $16 \mathrm{~S}$ rDNA and its adjacent ITS region were PCR amplified using the generic primer set pA (Edwards et al., 1989) and B23S (Lepère et al., 2000) as described in Rajaniemi et al. (2005). Gel purification of the amplified fragment was performed by using Aquapure genomic DNA extraction kit (Bio-Rad, Hercules, CA, USA), which was then cloned in pGEMT vector. After overnight incubation at $4^{\circ} \mathrm{C}$, plasmids were transformed into maximum efficiency competent cells (Life Technologies, Rockville, MD, USA) according to the manufacturer's instruction and plated onto LB Amp $\left(60 \mu \mathrm{g} \mathrm{mL} \mathrm{m}^{-1}\right)$ agar and incubated overnight at $37^{\circ} \mathrm{C}$. Sequencing was done by using ABI PRISM-3100 Genetic Analyzer (Applied Biosystems, Foster City, CA, USA) as described by Webb and Maas (2002).

\section{GENERATION OF ipdC KNOCKOUT MUTANTS IN Nostoc}

The putative ipdC gene was knocked out as described by Hussain etal. (2013). Genomic DNA was isolated from the cultures pre-incubated for 15 days by using UltraClean Soil DNA isolation Kit TM (Mo Bio Laboratories, Carlsbad, CA, USA) and following the instructions of the manufacturers. The putative ipdC gene was knocked out from the genome of Nostoc by replacing part of the gene sequence with a DNA fragment comprising of the sequence coding for kanamycin resistance (Homologous recombination). Open reading frame of putative $i p d C$ was PCR amplified from genomic DNA of Nostoc with IPDC-F 5'-AACGGCAAAAGTCACATTCC-3' and IPDC-R 5'-TGAGTTGACGAGGCAGTACG-3'. The amplified fragment was then ligated into the SmaI site of pUC19. A 169-bp fragment was dissected from the gene with Psil and ScaI, and replaced by a kanamycin resistance sequence conserved in pKRP11 by digestion with SmaI (Kaczmarzyk and Fulda, 2010). The construct was then used to transform Nostoc cells, as described by Summers et al. (1995). The suspension containing cells/DNA mixture was then plated on BG 11 medium (solidified with agarose) for the preliminary selection of transformants. After $24 \mathrm{~h}$ of incubation, kanamycin resistance of the transformants was tested by adding $0.45 \mathrm{mg}$ of kanamycin into the wells made in the agar plates. Homozygous mutant were obtained by successive streaking of the transformant on BG 11 plates supplemented with kanamycin. Complete segregation of the wild alleles and correct incorporation of the knockout cassette was validated by confirmatory PCR reactions. Primers used for complete segregation and validation of replacement of ipdC were: forward primer $5^{\prime}$-CTTCGGCACCAATTACGCAG-3' and reverse primer TTGCCCAACGAAAGTAGCTC-3'. The correct insertion of the kanamycin resistance cassette was confirmed by following Kaczmarzyk and Fulda (2010).

For genetic complementation of the putative ipdC mutant Nostoc, a modified version of the above mentioned knockout 
construct was used. In the modified construct, the kanamycin cassette was replaced by chloramphenicol resistance cassette which was released from the vector pKRP10 (Reece and Phillips, 1995). Fragments of Nostoc DNA, flanking the chloramphenicol cassette, served as borders for homologous recombination. EagI restriction site was introduced in flanking region at a distance $79 \mathrm{bp}$ from chloramphenicol cassette by changing 3 bp (QuikChange II site-directed mutagenesis kit, Stratagene) using $5^{\prime}$ GCTTTCTGCTTTAAGTCAACGGCCGCAG-3' and 5' CTGCGG CCGTTGACTTAAAGCAGAAAGC-3'). A 7433 bp fragment, including the open reading frame of putative ipdC gene of Nostoc with promoter and terminator, was amplified by PCR with forward and reverse primers (5'-GTAGCCCTGCGATCGCTATT3'and 5'-GATAGGGGCACCAGCAAAGT-3', respectively), harboring EagI restriction sites to both ends by using Expand Long Template PCR System (Roche Diagnostics) following the instructions of the manufacturer. The PCR amplified DNA fragment was, digested, cloned into the newly created EagI restriction site of the complementation vector and the resulting plasmid was used to transform Nostoc ipdC knockout mutant. Initial selection of transformants was made by spreading the DNA/cells mixture on solid BG 11 medium. Three evenly distributed wells were punched into the agar after $24 \mathrm{~h}$ to which $\mathrm{Cm}(0.45 \mathrm{mg})$ was added. Homozygous ipdC complemented Nostoc strains were obtained by successive streaking on BG 11 plates containing the antibiotic.

\section{DETERMINATION OF IAA IN Nostoc CULTURES}

Extraction and determination of IAA were performed as described by Hussain et al. (2010). Wild type, ipdC knockout mutant and ipdC complemented strains of Nostoc were grown separately in $250 \mathrm{~mL}$ liquid BG11 media containing $500 \mu \mathrm{g} \mathrm{mL}^{-1}$ of tryptophan at $25^{\circ} \mathrm{C}$ under a photoperiod of $8 \mathrm{~h}$ light $\left(18 \mathrm{lmol}\right.$ photons $\mathrm{m}^{-2}$ $\mathrm{s}^{-1}$ ) and $16 \mathrm{~h}$ dark for 3 weeks (inoculum density was adjusted to $0.2 \mu \mathrm{g} \mathrm{mL}^{-1} \mathrm{ch}-a$ ), respectively. Cellular as well as released IAA was measured in the cultures on a weekly basis for 3 weeks. For the extraction of IAA, cultures were harvested by centrifugation (16000 rpm for $5 \mathrm{~min}$ ) and the harvested supernatant was then filtered through nylon filters ( $22 \mu \mathrm{m}$ pore size). Bieleski buffer $(60 \%$ methanol, $25 \% \mathrm{CHCl}_{3}, 10 \% \mathrm{HCOOH}$, and $5 \% \mathrm{H}_{2} \mathrm{O}$ ) containing $10 \mathrm{pmol}$ [2H5] IAA as internal standard was used for the extraction of IAA separately from biomass and culture supernatant. After evaporation to dryness, the pooled extract was reconstituted in $5 \mathrm{~mL}$ of acidified water $(\mathrm{pH} 3)$ and was purified by solid phase extraction column (CHROMA-BOND ${ }^{\circledR}$ HR-XC, $3 \mathrm{ml}, 200 \mathrm{mg}$ ) following the manufacturer's instructions. The eluent was dried, re-dissolved in $15 \mathrm{mM}$ ammonium formate ( $\mathrm{pH} 4)$, and analyzed via UPLC-ESI-MS/MS under the conditions described by Hussain et al. (2010).

\section{ASSOCIATION OF Nostoc STRAINS WITH PLANT ROOTS}

Seeds of Triticum aestivum c.v. Uqab 2000 and Oriza sativa c.v. Basmati were surface-sterilized by shaking the seeds in $0.1 \% \mathrm{HgCl}_{2}$ solution for $5 \mathrm{~min}$. After draining out $\mathrm{HgCl}_{2}$ solution, the seeds were rinsed five times with sterile distilled water. Surface-sterilized seeds were then grown in Petri plates containing $7 \mathrm{~mL}$ half strength nutrient solution under axenic conditions. After germination,
$2 \mathrm{~mL}$ cyanobacterial suspension (adjusted to an inoculum density of $\left.1 \mu \mathrm{g} \mathrm{mL}{ }^{-1} \mathrm{ch}-a\right)$ was added to each plate and incubated for 2-weeks at $22 \pm 1{ }^{\circ} \mathrm{C}, 60 \%$ relative humidity, $12 \mathrm{~h}$ photoperiod, and light intensity adjusted to $180 \mu \mathrm{mol} \mathrm{m} \mathrm{m}^{-2} \mathrm{~s}^{-1}$. Seedlings were harvested after 2-weeks and the chlorophyll were extracted out of the roots with $80 \%$ methanol. Growth of Nostoc co-cultivated with rice seedlings was estimated by measuring the chlorophylla extracted from wild type or $\triangle i p d C$ Nostoc cells present in the culture media as well as those associated with plant roots. Chlorophyll- $a$ contents were determined by recording the OD of the samples at $660 \mathrm{~nm}$. Each treatment was replicated three times and the experiment was repeated twice.

\section{CHEMICAL COMPLEMENTATION WITH EXOGENOUS IAA}

Effect of exogenous IAA on the in vitro and in planta (colonization) growth of Nostoc was assessed by supplementing this compound in the culture media used to grow mutant Nostoc alone or in association with rice root, under growth conditions previously described. To reconstitute the concentration of IAA in mutant strain $(\triangle i p d C)$, IAA was supplemented to the culture media used for free grown or root associated Nostoc in concentration exactly equal to or twice as the difference in its concentration produced by wild type and mutant strains. Plate counting method was also used to count the number of free grown and root associated Nostoc for comparison with Chlorophyll-a estimation method. Dilutions of the culture of Nostoc and grounded root extracts were plated on agar plate to count the colony forming unit. Growth and colonization was estimated after incubation of 15 days.

\section{EXPRESSION OF ipdC IN PLANT ROOT ASSOCIATED Nostoc}

Quantitative RT-PCR was used to determine the expression of $i p d C$ gene in Nostoc associated with rice and wheat root and compared their expression with unassociated strains. Surface sterilized seeds of rice and wheat were grown as described above. After a week of germination, seedlings of each species were shifted to test tubes containing a suspension of Nostoc (adjusted to an inoculum density of $1 \mu \mathrm{g} \mathrm{mL}^{-1} \mathrm{ch}-a$ ) and incubated for 1 week under axenic conditions. Nostoc samples were collected after every $24 \mathrm{~h}$ for 1 week. From the root associated Nostoc, mRNA was isolated using UltraClean Plant RNA isolation kit (Mo Bio Laboratories), according to the manufacturer's instruction. To avoid any DNA or enzymes contamination, the RNA containing samples were treated with DNase I (Promega) followed by phenol-chloroform extraction and ethanol precipitation. Spectrophotometer (Nanodrop Technologies) was used to determine the quantity and quality of the RNA. Total RNA was resuspended in $7 \mu \mathrm{L}$ of deionized water and used as a template to synthesize cDNA following the protocol for First-strand cDNA synthesis (Fermentas) using random hexamers (Fermentas). The cDNA was used as a template for the expression analysis of $i p d C$ gene by qPCR using primers specific to this gene of Nostoc. For each time point (means for every $24 \mathrm{~h}$ ), two replicates from each treatment, i.e., free grown Nostoc, a negative control (without cDNA template), and a positive control (using Nostoc genomic DNA as a template) were analyzed. For each time point, the $\operatorname{rnpB}$ (the RNA component of RNase $\mathrm{P}$ ) was analyzed as a reference gene. The reaction mixture $(20 \mu \mathrm{L})$ for the qPCR was made by mixing $1 \mathrm{ng}$ of cDNA, 
$250 \mathrm{nM}$ of each specific primer, $10 \mu \mathrm{L} 2 \times$ SYBR green Supermix with Rox (Bio-Rad Laboratories, Inc.) and sterile water. Conditions of the qPCR were the same as described by Soule et al. (2009).

To confirm that each pair of primers will result in a single PCR product, a melting curve analysis was done. The levels of differential gene expression in extracts from the root-associated and free grown Nostoc were calculated as ratios from the $r n p B$ normalized real-time amplification data.

\section{PLANT GROWTH EXPERIMENTS}

Effect of different strains of Nostoc, wild type Os-1, $\Delta$ ipdC mutant, and $\triangle i p d C p i p d C$ complemented strains on the growth of rice and wheat was screened under axenic conditions. Surface sterilized seeds, described earlier, were soaked in $10 \mathrm{~mL}$ suspension of different strains of Nostoc (adjusted to an inoculum density of $1 \mu \mathrm{g} \mathrm{mL}^{-1} \mathrm{ch}-a$ ) separately. Nostoc loaded and control seeds (not treated with Nostoc) were then grown in pots (three seeds per pot) containing sterilized garden soil under axenic conditions for 2 weeks at $22 \pm 1{ }^{\circ} \mathrm{C}, 60 \%$ relative humidity, $12 \mathrm{~h}$ photoperiod, and light intensity adjusted to $180 \mu \mathrm{mol} \mathrm{m}^{-2} \mathrm{~s}^{-1}$. After harvesting the seedlings, different growth parameters including shoot length, root length, fresh, and dry weight were recorded. Each treatment was replicated three times and the experiment was repeated three times.

\section{STATISTICAL ANALYSIS}

The data was analyzed statistically by performing one way or three-way analysis of variance (ANOVA) and Duncan multiple range test at $p=0.05$, using SPSS for windows (Version 20.0; SPSS Inc., Chicago, IL, USA). Two samples Student's $t$-test was also used to check significance of the data obtained in Nostoc growth experiment (Microsoft Excel 2013).

\section{RESULTS}

\section{SCREENING AND IDENTIFICATION OF THE ISOLATES}

Three randomly selected plants each of wheat and rice were uprooted and brought to the lab. Three Petri plates were inoculated with four pieces of roots from each plant. Of 36 root pieces processed separately from rice and wheat, only five and two cyanobacterial isolates were found, which indicated the colonization percentage of rice $(13.9 \%)$ and wheat $(5.5 \%)$, respectively, (Table 1). Whereas, endophytic Nostoc was found only in rice roots. Growth of cyanobacterial colonies started on the 6th day of the incubation of root segments on BG11 agar plates and the majority of the isolates (4 out of 7) were identified as Nostoc when their morphology was studied under a light microscope. IAA analysis revealed that Nostoc (Os-1) strain was the only one to synthesize IAA among the various Nostoc isolates (Table 1). The identity of the strain was further confirmed by assessing the homology of the partial sequence of its 16S-23S rDNA intergenic spacer region and the sequence was deposited in NCBI GenBank (accession number KP001508).

\section{EXPRESSION OF ipdC IN ROOTS ASSOCIATED Nostoc}

To correlate the production of IAA with the expression of IAA biosynthesis genes, we first determine the expression of putative
Table 1 | Strains of cyanobacteria isolated from roots of wheat and rice.

\begin{tabular}{lllll}
\hline Strains & Source & Identification & $\begin{array}{l}\text { IAA } \\
\text { (colorimetric) }\end{array}$ & $\begin{array}{l}\text { Putative } \\
\text { ipdC by } \\
\text { PCR }\end{array}$ \\
\hline Os-1 & Oryza sativa L. & Nostoc & $22 \pm 1.2$ & Yes \\
Os-2 & Oryza sativa L. & Phormidium & $1 \pm 0.05$ & No \\
Os-3 & Oryza sativa L. & Nostoc & BDL & No \\
Os-4 & Oryza sativa L. & Nostoc & BDL & No \\
Os-5 & Oryza sativa L. & Nostoc & $1.3 \pm 0.1$ & No \\
Te-1 & Triticumaestivum L. & Anabaena & $31 \pm 1.7$ & No \\
Te-2 & Triticumaestivum L. Anabaena & $17 \pm 1.2$ & No \\
\hline
\end{tabular}

$B D L$, below detection limit

ipdC gene in free and rice roots associated Nostoc (grown under similar conditions) was measured by qPCR method and was compared. At time zero, expression of ipdC gene was similar in both root-associated and free living Nostoc (Figure 1). After $24 \mathrm{~h}$, the ipdC expression was threefold greater in the root associated Nostoc sp. Os-1 compared to free grown Os-1 strain (Figure 1). In the following hours a fivefold increase in ipdC expression between root associated and free grown Nostoc sp. Os-1 was observed. The significant expression of putative ipdC gene in root-associated Nostoc strain led us to examine the involvement of this gene in root colonization by the cyanobacterium.

\section{GENERATION OF ipdC KNOCKOUT MUTANTS IN Nostoc}

To investigate the biological function of IAA and potential involvement of ipdC in IAA production and root colonization, an ipdC knockout mutant was generated. A 169 bp of the coding region of the putative $i p d C$ was replaced with a kanamycin resistance cassette to inactivate the gene. Since many copies of chromosomes can be found in a single cell of Nostoc (Jain et al., 2012), it was imperative

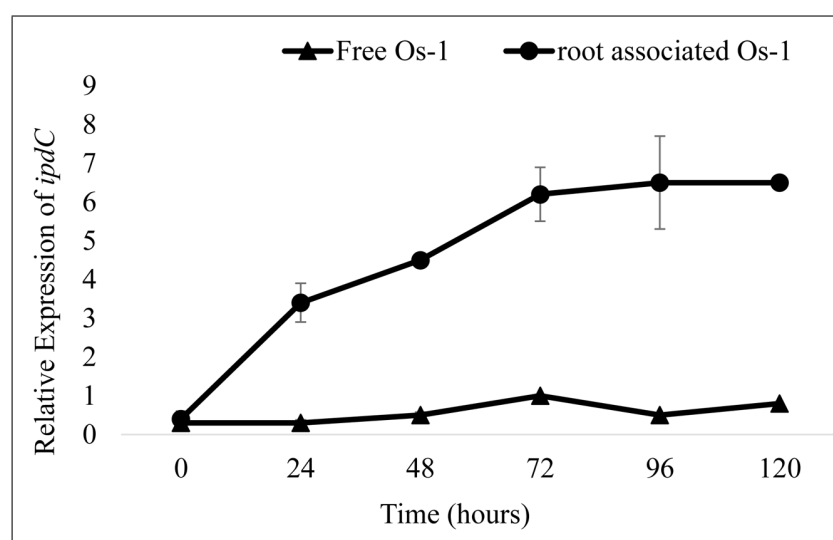

FIGURE 1 | Gene expression dynamics of putative ipdC genes of Nostoc in response to root colonization, based on qPCR of reverse-transcribed RNA extracts. Relative expression of ipdC in Nostoc Os-1 grown alone (Free Os-1) or colonized on rice root (root associated Os-1). Graph represents data from three replicates in two independent experiments (means $\pm \mathrm{SD} ; n=6$ ). 
to monitor the complete segregation of the wild allele by PCR. The knockout of the ipdC gene was confirmed by no amplification of ipdC specific primers by PCR. Using genomic DNA of Nostoc Os1 (wild type strain), a fragment of $150 \mathrm{bp}$ was amplified with the same pair of primers confirming the validity of the primers. Successful insertion with the right orientation of the fragment containing the sequence for resistance against kanamycin within chromosome of ipdC mutant strain $(\Delta i p d C)$, was confirmed by using kanamycin specific primers. The result of these two PCR reactions indicated removal of the functional ipdC gene in the mutant Nostoc strains.

\section{GROWTH ANALYSIS OF WILD TYPE, ipdC MUTANT, AND COMPLEMENTED Nostoc STRAINS}

To determine whether the deletion of ipdC affects Nostoc growth, we first analyze the growth of Nostoc by measuring chlorophyll- $a$ content, which has been an acceptable method to quantify Nostoc growth and colonization in plant roots by Nilsson et al. (2002) and Hussain et al. (2013). Wild type (Os-1), ipdC knockout mutant $(\triangle i p d C)$, and complemented strain ( $\triangle i p d C p i p d C)$ strains of Nostoc were cultured in vitro in BG11 medium at various $\mathrm{pH}$ and temperature. As shown in Figure 2, we found that the growth of wild type Os-1 was significantly greater than the growth of $i p d C$ knockout mutant in all growth conditions tested $(p<0.05)$. Importantly, the complementation strain $\Delta i p d C p i p d C$ showed significant improvement in its growth (Figure 2). To determine if Nostoc growth was also compromised when it was grown in culture medium co-cultivated with rice seedlings in Petri plate, the Nostoc cells were collected and determined for the amount of chlorophyll- $a$ after co-cultivated with rice seedlings for 15 days. There was no significant difference in the growth of wild type and $\Delta i p d C$ mutant when grown in this way (Figure 3).

\section{PHYTOHORMONES DETERMINATION}

An optimum condition of $25^{\circ} \mathrm{C}, \mathrm{pH} 8.0$, and photoperiod of 8 light and $16 \mathrm{~h}$ dark were kept for growth and production of IAA

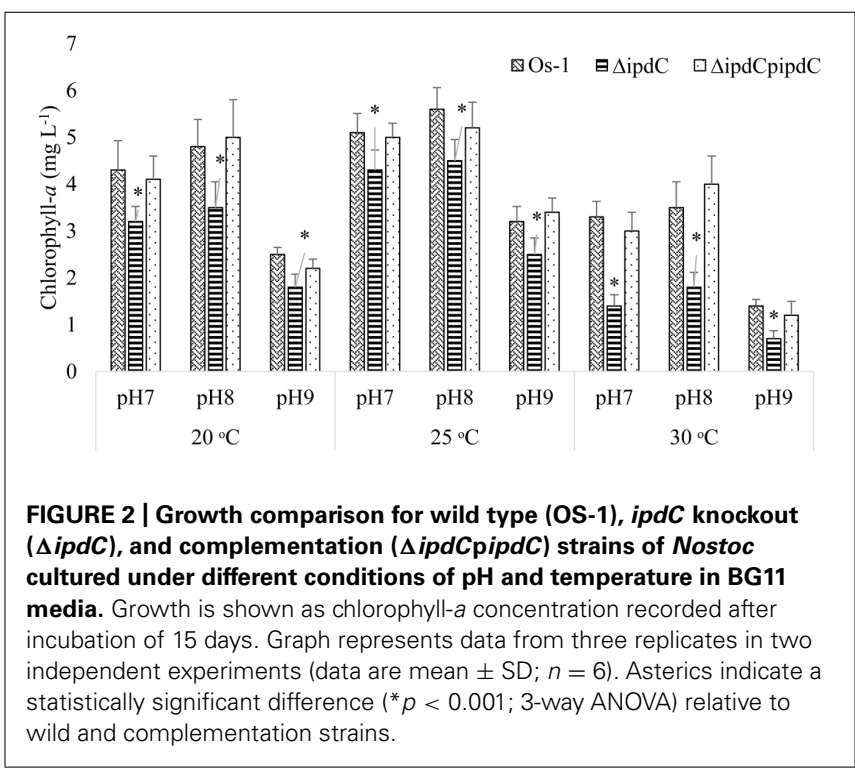

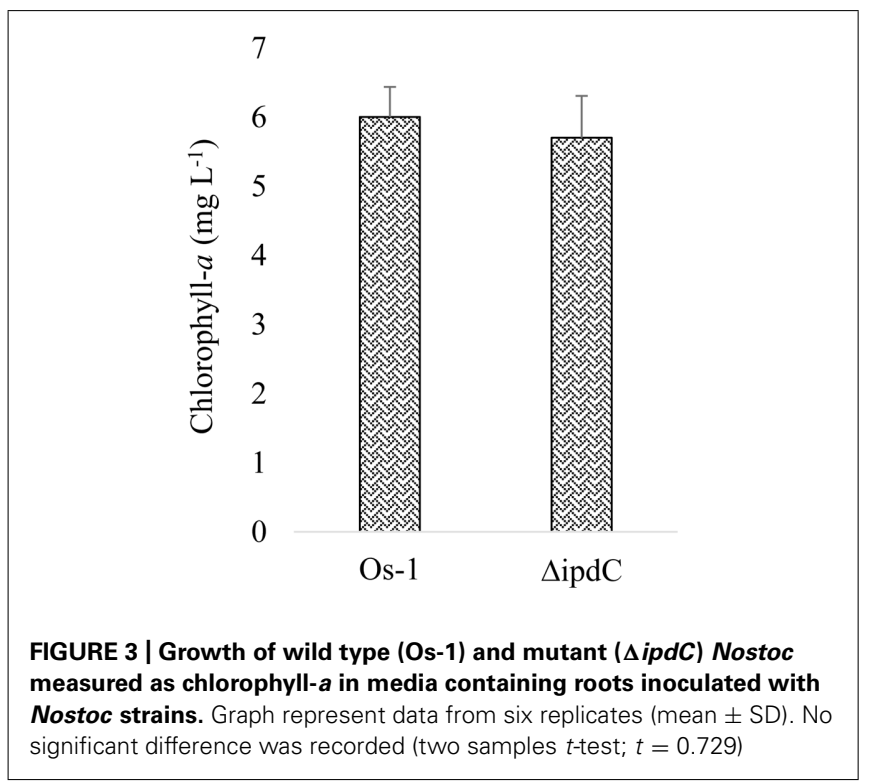

by Nostoc. The wild-type strain of Nostoc released not only a significant amount of IAA in the culture media, but also retained IAA in the filaments under the set conditions. However, amount of IAA released was always greater than the amount of IAA retained in the filaments (Figure 4). The difference was increased sharply with increasing incubation time and reached to maximum (i.e., $78 \%$ ) during the third week of incubation (Figure 4).

Also the amount of IAA peaked in culture incubated for 3 weeks in a medium with optimal amount of tryptophan $\left(500 \mu \mathrm{g} \mathrm{mL}^{-1}\right)$, higher amount of tryptophan was detrimental for the growth of Nostoc. After 3 weeks of incubation, a 78\% reduction in the production of IAA (from $34.2 \mathrm{pmol} \mathrm{mg}^{-1}$ of ch- $a$ to $7.6 \mathrm{pmol}$ $\mathrm{mg}^{-1}$ of ch- $a$ ) was documented in the culture of mutant strain compared to wild type strain OS-1 (Figure 4). IAA to Biomass ratio in tryptophan supplemented media was also reduced by 
more than fourfold in $\triangle i p d C$ than that of wild type strain. The $\triangle i p d C$ pipdC complementation strain restored wild-type level of IAA production. The results suggested that $i p d C$ plays a role for IAA production, in which the amount was increased with time and in the presence of tryptophan (Figure 4).

\section{COLONIZATION OF Nostoc ON PLANT ROOTS}

Wild type strain of Nostoc efficiently colonized in the roots of rice as well as wheat seedlings. In the absence of Nostoc, no chlorophyll- $a$ was detected in the roots of the selected plants. A gradual increase in the concentration of chlorophyll- $a$ was recorded in the Nostoc colonized roots, as an indication of an increase in the growth of root-associated Nostoc. Concentration of chlorophyll- $a$ in colonized root of rice and wheat by Nostoc Os-1 was increased from 14 to $12 \mu \mathrm{g} \mathrm{g}^{-1} \mathrm{FW}$ (at the end of the first week) to 38 and $28 \mu \mathrm{g} \mathrm{g}^{-1} \mathrm{FW}$ (by the end of the third week; Figure 5). We noticed that $i p d C$ knockout strain $\triangle i p d C$ (low IAA mutant) was less efficient to colonize in the roots of rice as well as wheat seedlings, which is correlated with the reduced amounts of IAA (Figure 5). After co-cultivation of 1 week, significantly less amount (59\% of the wild type strain) of $\triangle i p d C$ mutant was colonized in wheat root. By the end of second and third week, the amount of chlorophyll- $a$ in the roots of wheat colonized by the mutant strain was 39 and $40 \%$ lower than that of the roots colonized by wild-type strain. For rice, the colonization efficiency $\triangle i p d C$ was only $43 \%$ of the wild-type strain of Nostoc during first week. In the subsequent weeks, the root chlorophyll- $a$ was up to $40.9 \%$ lower in root colonized by the mutant as compared to the wild-type strain. The root colonization efficiency of ipdC knockout mutant was restored to wild type level by complementation (Figure 5).

\section{EXOGENOUS APPLICATION OF IAA RESTORED COLONIZATION EFFICIENCY OF THE $\triangle$ IPdC MUTANT}

When applied exogenously, IAA restored the in vitro growth as well as colonization efficiency of the mutant Nostoc when the concentration of chlorophyll- $a$ was used as an indication of Nostoc growth

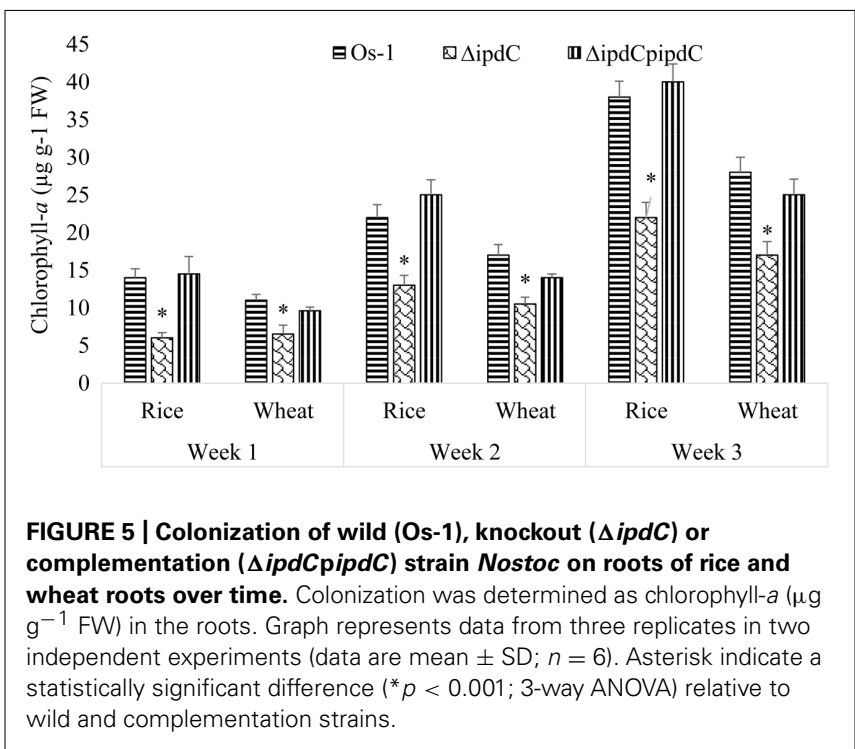

(Figure 6). To confirm the correlation between chlorophyll-a concentration and plate counting method for cyanobacterial growth, colony-forming-unit (cfu) was also used to determine Nostoc growth. We showed that both chlorophyll- $a$ and plate counting assays revealed the reduced root colonization efficiency of the $\Delta i p d C$ mutant strain (Figure 6). However, cfu of $\Delta i p d C$ was similar to that of wild type strain, which is inconsistent with the growth assay estimated by chlorophyll- $a$ amounts.

\section{PHYTOSTIMULATION UNDER AXENIC CONDITIONS}

We also determined the impacts of $i p d C$ gene in plant growth. As shown in Table 2, seedlings of rice and wheat, inoculated with Nostoc sp. Os-1 (wild type strain) showed significantly greater growth than their respective controls. An overall increase of 23.5 and $50 \%$ in weight on dry and fresh weight basis was observed in wheat seedlings associated with Nostoc Os-1 as compared to the control. A similar response of rice to colonization by Nostoc was demonstrated. The effect of colonization on other growth parameters such as shoot length and root length of wheat and rice

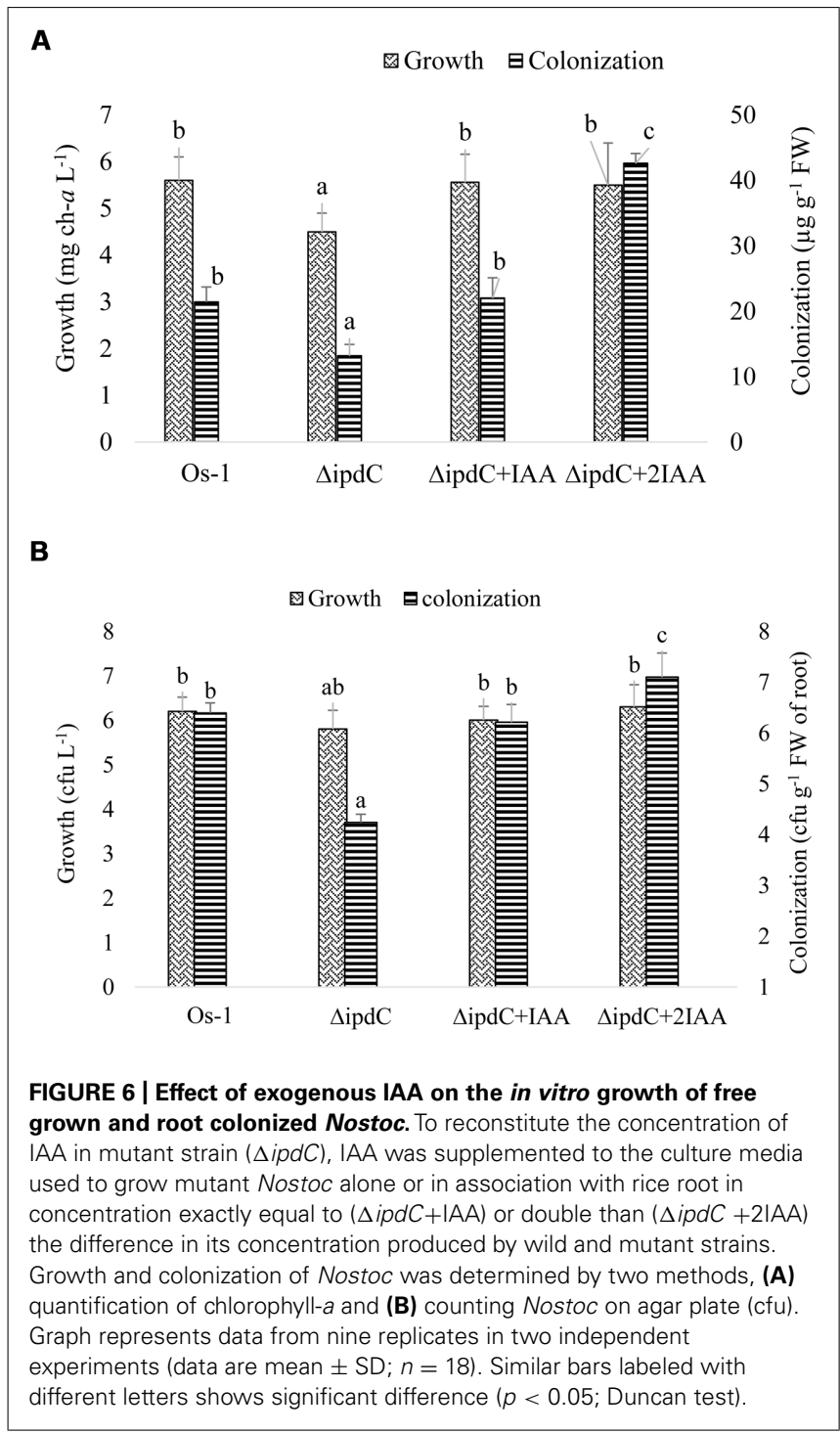


Table 2 | Growth parameters of wheat and rice seedlings recorded after growth of 2 weeks under axenic conditions.

\begin{tabular}{|c|c|c|c|c|c|c|c|c|}
\hline \multirow[t]{2}{*}{ Strain } & \multicolumn{4}{|c|}{$\mathbf{c m}$} & \multicolumn{4}{|c|}{ mg plant $^{-1}$} \\
\hline & \multicolumn{2}{|c|}{ Shoot length } & \multicolumn{2}{|c|}{ Root length } & \multicolumn{2}{|c|}{ Fresh weight } & \multicolumn{2}{|c|}{ Dry weight } \\
\hline Control & 13.2 (a) & 8.1 (a) & 3.8 (a) & 3.0 (a) & 1.8 (a) & $1.3(a)$ & 0.34 (a) & 0.17 (a) \\
\hline Os-1 & 16.4 (c) & 11.0 (c) & 6.5 (c) & $5.4(b)$ & 2.7 (b) & 1. 9 (b) & $0.42(b)$ & 0.25 (b) \\
\hline$\Delta i p d C$ pipdC & 15.5 (c) & 12 (c) & $5.8(c)$ & 4.9 (b) & $2.8(b)$ & $2.0(b)$ & 0.36 (a) & $0.23(b)$ \\
\hline
\end{tabular}

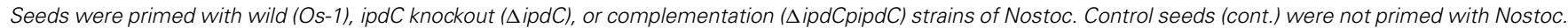
Different letters in the same column indicate significant difference between means (Duncan Multiple Range Test; $p<0.05$ ).

seedlings was remarkable. On the other hand, seedlings associated with mutant strain $\triangle i p d C$ grew similarly or only slightly better than their respective controls (no infection), suggesting the role of colonization and IAA in plant growth promotion by Nostoc (Table 2). However, complementation of wild-type ipdC restored the phytostimulatory efficiency of the knockout strain of Nostoc.

\section{DISCUSSION}

Successful colonization on root is a key to plant growth promotion by cyanobacteria (Karthikeyan et al., 2007). Being renowned for its symbiotic association with plants, Nostoc was chosen to study their efficacy of colonization on plant roots as affected by self-produced IAA. A simple approach of chlorophyll- $a$ determination in plant roots by Nilsson et al. (2002) and Hussain et al. (2013) was adopted to quantify root colonization by Nostoc. Under axenic conditions, the strains of Nostoc were able to produce IAA that enhanced their ability to colonize on wheat and rice roots. Also the induction of putative ipdC gene, a key gene of the IAA biosynthesis pathway from Nostoc (Sergeeva et al., 2002), suggested the involvement of IAA in the phenomenon, which is comparable to the findings of Tadra-Sfeir et al. (2011). It has been demonstrated that IAA production increases in Rhizobium when exposed to plant signals, the flavonoids, and IAA in turn enhances the release of flavonoids in root exudates of maize plant (Theunis et al., 2004; Dardanelli et al., 2008). To elaborate on the validity of the concept, ipdC gene was knocked out from Nostoc by homologous recombination. Significant loss in the ability of Nostoc to colonize on plant roots after knocking out ipdC provided an evidence of the role of IAA in plant-Nostoc interaction. IAA has been implicated in root colonization by rhizobacteria and fungi (Karabaghli-Degron et al., 1998; Lambrecht et al., 2000). Similarly, exogenous application of synthetic auxin, 2,4-dichlorophenoxyacetic acid (2,4-D), on wheat roots led to the formation of tumor-like structures, para-nodules, which were readily colonized by Nostoc (Gantar and Elhai, 1999). They had recorded up to 3.6 times more Nostoc on 2,4-D treated roots compared to untreated roots. Root colonization efficiency of IAA delivering non-heterocystous cyanobacteria was constantly more noteworthy than those that fail to offer this capacity (Ahmed et al., 2010). Besides 2,4-D, Cytokinins (Cks) are also used by Nostoc to colonize on roots (Hussain et al., 2013). It seems that auxin and Cks interact synergistically to promote root colonization by microbes. Synergistic interaction between
Cks and auxin also exists in nodule organogenesis (Hwang et al., 2012). However, direct evidence of the involvement of ipdC or IAA in root colonization by Nostoc is reported for the first time. Our work was a validation of the previous speculations about the possible role of IAA in root colonization by cyanobacteria Nostoc (Nilsson et al., 2002). Indeed root colonization capability of ipdC knockout strain was reduced up to $41 \%$ and was not lost totally, showing the inclusion of elements other than IAA in this process. Furthermore, impact of knocking out ipdC from Nostoc was more intensive on its capacity to produce IAA as compared with its capability to colonize on plant root. Loss of ipdC gene from Nostoc was accompanied by a decline in its potential to promote plant development, consistent with previous conclusions that microbial-produced IAA has the potential to promote plant growth (Hussain and Hasnain, 2011). Reduction in the growth of ipdC knockout Nostoc was a validation of the previous study reporting the imperative part of IAA in cyanobacterial growth and development (Leganés et al., 1987).

Reduction in growth of Nostoc due to loss of ipdC gene suggested that this gene product was important for optimal growth of Nostoc. Thus, lower root colonization efficiency of the ipdC knockout mutant could be due to its compromised fitness rather than involving ipdC or IAA directly. Similar growth of wild type and $\triangle i p d C$ Nostoc in culture medium co-cultivated with plant roots suggested the role of IAA in root colonization. Additionally, the evidence that the in vitro growth, root colonization efficiency, and plant growth promoting activity of the ipdC mutant strain could be restored when IAA was exogenously applied in the culture medium also suggested the involvement of IAA in root colonization and plant promoting efficiency of Nostoc.

Though chlorophyll- $a$ determination is an acceptable method used by Nilsson et al. (2002) and Hussain et al. (2013) to quantify root colonization by Nostoc, chlorophyll- $a$ amounts may be an indicative of the overall cell growth instead of the number of cyanobacterial cells or filaments. Thus, colony forming units (cfu) of free grown and root-associated Nostoc were counted on agar plate. While both chlorophyll- $a$ and plate counting assays revealed the reduced root colonization efficiency of the $\triangle i p d C$ mutant strain, reduced chlorophyll- $a$ in $\triangle i p d C$ during in vitro growth was not confirmed by plate counting analysis. These results indicated that chlorophyll- $a$ concentration is not necessarily correlated with cyanobacterial growth. These results 
suggested that the reduction of chlorophyll-a in the in vitro grown mutant strain was not due to the reduced growth but was due to reduction in chlorophyll-a synthesis/accumulation. If so, it is plausible to suggest a role of IAA in chlorophyll-a synthesis by Nostoc. Stimulatory effect of IAA on chlorophyll- $a$ and growth of Spirulina has been observed (Mohammed and Mohd, 2011). Another possibility is that IAA might affect hormogonia production (Hunter and Provasoli, 1964), which is necessary for root colonization as well as growth of cyanobacteria. Also, plate counting may result in over estimation of Nostoc due to filament breakage or hormogonia formation. Similarly, incomplete separation of the filaments in clusters may lead to under estimation of cell number (Whitton, 2002). To this end, we could not exclude the possibility that the compromised growth of $\Delta i p d C$ mutant may indirectly lead to its reduced colonization on plant root. However, the induction of ipdC gene expression upon its association with roots and the correlation of IAA production with root colonization efficiency and plant growth promoting activity indeed supported a role of IAA in root colonization by Nostoc.

Our findings highlighted the important role of IAA in root colonization by Nostoc. Restoration of root colonization ability of an ipdC mutant strain by exogenous IAA addition further confirmed the role of IAA in plant-Nostoc interaction. It may be concluded that IAA plays a vital role in root colonization by Nostoc and consequently plant growth promotion. Additionally, Nostoc's own growth and development may be affected by IAA.

\section{REFERENCES}

Adams, D. G., and Duggan, P. S. (2012). "Signalling in cyanobacteria-plant symbioses," in Signaling and Communication in Plant Symbiosis, Vol. 11, eds S. Perotto and F. Balauška (Berlin Heidelberg: Springer) 11, 93-121. doi: 10.1007/978-3-642-20966-6_5

Ahmed, M., Stal, L. J., and Hasnain, S. (2010). Association of non-heterocystous cyanobacteria with crop plants. Plant Soil 336, 363-375. doi: 10.1007/s11104010-0488-x

Akashi, H., and Gojobori, T. (2002). Metabolic efficiency and amino acid composition in the proteomes of Escherichia coli and Bacillus subtilis. Proc. Natl. Acad. Sci. U.S.A. 99, 3695-3700. doi: 10.1073/pnas.062526999

Barker, S. J., and Tagu, D. (2000). The roles of auxins and cytokinins in mycorrhizal symbioses. J. Plant Growth Regul. 19, 144-154.

Berg, G. (2009). Plant-microbe interactions promoting plant growth and health: perspectives for controlled use of microorganisms in agriculture. Appl. Microbiol. Biotechnol. 84, 11-18. doi: 10.1007/s00253-009-2092-7

Boopathi, T., Balamurugan, V., Gopinath, S., and Sundararaman, M. (2013). Characterization of IAA production by the mangrove cyanobacterium Phormidium sp. MI405019 and its influence on tobacco seed germination and organogenesis. J. Plant Growth Regul. 32, 758-766. doi: 10.1007/s00344-01 3-9342-8

Bossuyt, S., Spaepen, S., and Vanderleyden, J. (2012). Role of Auxin Signaling in the Interaction of Arabidopsis with the Plant Growth-Promoting Bacterium Azospirillum. Roots to the Future. Dundee.

Brandl, M. T., and Lindow, S. E. (1997). Environmental signals modulate the expression of an indole-3-acetic acid biosynthetic gene in Erwinia herbicola. Mol. Plant Microbe Interact. 10, 499-505. doi: 10.1094/MPMI.1997.10.4.499

Bruto, M., Prigent-Combaret, C., Muller, D., and Moënne-Loccoz, Y. (2014). Analysis of genes contributing to plant-beneficial functions in plant growth-promoting rhizobacteria and related Proteobacteria. Sci. Rep. 4:6261. doi: 10.1038/srep06261

Buick, R. (2008). When did oxygenic photosynthesis evolve? Philos. Trans. R. Soc. Lon. B Biol. Sci. 363, 2731-2743. doi: 10.1098/rstb.2008.0041

Costacurta, A., Keijers, V., and Vanderleyden, J. (1994). Molecular cloning and sequence analysis of an Azospirilium brasilense indole-3-pyruvate decarboxylase gene. Mol. Gen. Genet. 243, 463-472.
Dardanelli, M. S., Fernández De Córdoba, F. J., Espuny, M. R., Rodríguez Carvajal, M. A., Soria Díaz, M. E., Gil Serrano, A. M., et al. (2008). Effect of Azospirillum brasilense coinoculated with Rhizobium on Phaseolus vulgaris flavonoids and nod factor production under salt stress. Soil Biol. Biochem. 40, 2713-2721. doi: 10.1016/j.soilbio.2008.06.016

Edwards, U., Rogall, T., Blöcker, H., Emde, M., and Böttger, E. C. (1989). Isolation and direct complete nucleotide determination of entire genes. Characterization of a gene coding for $16 \mathrm{~S}$ ribosomal RNA. Nucleic Acids Res. 17, 7843-7853. doi: 10.1093/nar/17.19.7843

Gantar, M., and Elhai, J. (1999). Colonization of wheat para-nodules by the N2fixing cyanobacterium Nostoc sp. strain 2S9B. New phytol. 141, 373-379. doi: 10.1046/j.1469-8137.1999.00352.x

Hunter, S. H., and Provasoli, L. (1964). Nutrition of algae. Annu. Rev. Plant Physiol. 15, 37-56. doi: 10.1146/annurev.pp.15.060164.000345

Hussain, A., Hamayun, M., and Shah, S. T. (2013). Root colonization and phytostimulation by phytohormones producing entophytic Nostoc sp. AH-12. Curr. Microbiol. 67, 624-630. doi: 10.1007/s00284-013-0408-4

Hussain, A., and Hasnain, S. (2011). Phytostimulation and biofertilization in wheat by cyanobacteria. J. Ind. Microbiol. Biotechnol. 38, 85-92. doi: 10.1007/s10295010-0833-3

Hussain, A., Krischke, M., Roitsch, T., and Hasnain, S. (2010). Rapid determination of cytokinins and auxin in cyanobacteria. Curr. Microbiol. 61, 361-369. doi: 10.1007/s00284-010-9620-7

Hwang, I., Sheen, J., and Müller, B. (2012). Cytokinin signaling networks. Annu. Rev. Plant Biol. 63, 353-380. doi: 10.1146/annurev-arplant-042811-105503

Jain, I. H., Vijayan, V., and O'Shea, E. K. (2012). Spatial ordering of chromosomes enhances the fidelity of chromosome partitioning in cyanobacteria. Proc. Natl. Acad. Sci. U.S.A. 109, 13638-13643. doi: 10.1073/pnas.1211 144109

Kaczmarzyk, D., and Fulda, M. (2010). Fatty acid activation in cyanobacteria mediated by acyl-acyl carrier protein synthetase enables fatty acid recycling. Plant Physiol. 152, 1598-1610. doi: 10.1104/pp.109.148007

Karabaghli-Degron, C., Sotta, B., Bonnet, M., Gay, G., and Le Tacon, F. (1998). The auxin transport inhibitor 2, 3, 5-triiodobenzoic acid (TIBA) inhibits the stimulation of in vitro lateral root formation and the colonization of the tap-root cortex of Norway spruce (Piceaabies) seedlings by the ectomycorrhizal fungus Laccaria bicolor. New Phytol. 140, 723-733. doi: 10.1046/j.1469-8137.1998.00307.x

Karthikeyan, N., Prasanna, R., Nain, L., and Kaushik, B. D. (2007). Evaluating the potential of plant growth promoting cyanobacteria as inoculants for wheat. Eur. J. Soil Biol. 43, 23-30. doi: 10.1016/j.ejsobi.2006.11.001

Koga, J., Adachi, T., and Hidaka, H. (1991). Molecular cloning of the gene for indolepyruvate decarboxylase from Enterobacter cloacae. Mol. Gen. Genet. 226, 10-16. doi: 10.1007/BF00273581

Lambrecht, M., Okon, Y., VandeBroek, A., and Vanderleyden, J. (2000). Indole-3acetic acid: a reciprocal signalling molecule in bacteria-plant interactions. Trends Microbiol. 8, 298-300. doi: 10.1016/S0966-842X(00)01732-7

Leganés, F., Sánchez-Maeso, E., and Fernández-Valiente, E. (1987). Effect of indoleacetic acid on growth and dinitrogen fixation in cyanobacteria. Plant Cell Physiol. 28, 529-533.

Lepère, C., Wilmotte, A., and Meyer, B. (2000). Molecular diversity of Microcystis strains (Cyanophyceae, Chroococcales) based on 16S rDNA sequences. Syst. Geogr. Plants 70, 275-283. doi: 10.2307/3668646

Mohammed, M. K., and Mohd, M. K. (2011). Production of carotenoids (antioxidants/colourant) in Spirulina platensis in response to indole acetic acid (IAA). Int. J. Eng. Sci. Technol. 3, 4973-4979.

Myllys, L., Stenroos, S., Thell, A., and Kuusinen, M. (2007). High cyanobiont selectivity of epiphytic lichens in old growth boreal forest of Finland. New phytol. 173, 621-629. doi: 10.1111/j.1469-8137.2006.01944.x

Nilsson, M., Bhattacharya, J., Rai, A. N., and Bergman, B. (2002). Colonization of roots of rice (Oryza sativa) by symbiotic Nostoc strains. New phytol. 156, 517-525. doi: 10.1046/j.1469-8137.2002.00534.x

Olsson, S., Kaasalainen, U., and Rikkinen, J. (2012). Reconstruction of structural evolution in the trnL intron P6b loop of symbiotic Nostoc (cyanobacteria). Curr. Genet. 58, 49-58. doi: 10.1007/s00294-011-0364-0

Pankratova, E. M., Trefilova, L. V., Zyablykh, R. Y., and Ustyuzhanin, I. A. (2008). Cyanobacterium Nostoc paludosumKütz as a basis for creation of agriculturally useful microbial associations by the example of bacteria of the genus Rhizobium. Microbiology 77, 228-234. doi: 10.1134/S00262617080 20173 
Patten, C. L., Blakney, A. J. C., and Coulson, T. J. D. (2013). Activity, distribution and function of indole-3-acetic acid biosynthetic pathways in bacteria. Crit. Rev. Microbiol. 39, 395-415. doi: 10.3109/1040841X.2012. 716819

Prinsen, E., Costacurta, A., Michiels, K., Vanderleyden, J., and Van Onckelen, H. (1993). Azospirillum brasilense indole-3-acetic acid biosynthesis: evidence for a non-tryptophan dependent pathway. Mol. Plant Microbe Interact. 6, 609-609. doi: 10.1094/MPMI-6-609

Rai, A. N., and Bergman, B. (2000). "Creation of new nitrogen-fixing cyanobacterial associations (special issue - biology and environment)," in Proceedings of the Royal Irish Academy, Vol. 102B (Dublin: Royal Irish Academy), 65-68.

Rajaniemi, P., Hrouzek, P., Kaštovská, K. r., Willame, R., Rantala, A., Hoffmann, L., etal. (2005). Phylogenetic and morphological evaluation of the genera Anabaena, Aphanizomenon, Trichormus, and Nostoc (Nostocales, Cyanobacteria). Int. J. Syst. Evol. Microbiol. 55, 11-26. doi: 10.1099/ijs.0. 63276-0

Reece, K. S., and Phillips, G. J. (1995). New plasmids carrying antibioticresistance cassettes. Gene 165, 141-142. doi: 10.1016/0378-1119(95) 00529-F

Schütz, A., Sandalova, T., Ricagno, S., Hübner, G., König, S., and Schneider, G. (2003). Crystal structure of thiamindiphosphatedependent indolepyruvate decarboxylase from Enterobacter cloacae, an enzyme involved in the biosynthesis of the plant hormone indole-3-acetic acid. Eur. J. Biochem. 270, 2312-2321. doi: 10.1046/j.1432-1033.2003. 03601.x

Sergeeva, E., Liaimer, A., and Bergman, B. (2002). Evidence for production of the phytohormone indole-3-acetic acid by cyanobacteria. Planta 215, 229-238. doi: 10.1007/s00425-002-0749-x

Soule, T., Garcia-Pichel, F., and Stout, V. (2009). Gene expression patterns associated with the biosynthesis of the sunscreen scytonemin in Nostoc punctiforme ATCC 29133 in response to UVA radiation. J. Bacteriol. 191, 4639-4646. doi: 10.1128/JB.00134-09

Summers, M. L., Wallis, J. G., Campbell, E. L., and Meeks, J. C. (1995). Genetic evidence of a major role for glucose-6-phosphate dehydrogenase in nitrogen fixation and dark growth of the cyanobacterium Nostoc sp. strain ATCC 29133. J. Bacteriol. 177, 6184-6194.
Tadra-Sfeir, M. Z., Souza, E. M., Faoro, H., Müller-Santos, M., Baura, V. A., Tuleski, T. R., et al. (2011). Naringenin regulates expression of genes involved in cell wall synthesis in Herbaspirillum seropedicae. Appl. Environ. Microbiol. 77, 2180-2183. doi: 10.1128/AEM.02071-10

Theunis, M., Kobayashi, H., Broughton, W. J., and Prinsen, E. (2004). Flavonoids, NodD1, NodD2, and nod-box NB15 modulate expression of the y4wEFG locus that is required for indole-3-acetic acid synthesis in Rhizobium sp. strain NGR234. Mol. Plant Microbe Interact. 17, 1153-1161. doi: 10.1094/MPMI.2004.17.10.1153

Webb, V. L., and Maas, E. W. (2002). Sequence analysis of 16S rRNA gene of cyanobacteria associated with the marine sponge Mycale (Carmia) hentscheli. FEMS Microbiol. Lett. 207, 43-47. doi: 10.1111/j.1574-6968.2002.tb11026.x

Whitton, B. A. (2002). "Soil and rice-fields," in The Ecology of Cyanobacteria, eds B. A. Whitton and M. Potts (Dordrecht: Kluwer Academic Publishers), 233-255. doi: 10.1007/0-306-46855-7 8

Zhang, Z., Li, Q., Li, Z., Staswick, P. E., Wang, M., Zhu, Y., et al. (2007). Dual regulation role of GH3. 5 in salicylic acid and auxin signaling during Arabidopsis-Pseudomonas syringae interaction. Plant Physiol. 145, 450-464. doi: $10.1104 / \mathrm{pp} .107 .106021$

Conflict of Interest Statement: The authors declare that the research was conducted in the absence of any commercial or financial relationships that could be construed as a potential conflict of interest.

Received: 23 August 2014; accepted: 16 January 2015; published online: 05 February 2015.

Citation: Hussain A, Shah ST, Rahman H, Irshad M and Iqbal A (2015) Effect of IAA on in vitro growth and colonization of Nostoc in plant roots. Front. Plant Sci. 6:46. doi: $10.3389 / \mathrm{fpls} .2015 .00046$

This article was submitted to Plant-Microbe Interaction, a section of the journal Frontiers in Plant Science.

Copyright (c) 2015 Hussain, Shah, Rahman, Irshad and Iqbal. This is an openaccess article distributed under the terms of the Creative Commons Attribution License (CC BY). The use, distribution or reproduction in other forums is permitted, provided the original author(s) or licensor are credited and that the original publication in this journal is cited, in accordance with accepted academic practice. No use, distribution or reproduction is permitted which does not comply with these terms. 